\title{
Enfoque ortodóncico en el rratamiento multidisciplinario de pacientes adultos su relación con implantes y prostodoncia
}

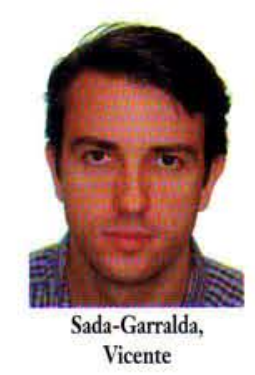

\author{
Orthodontic approach in multidisciplinary treatment of adult patients \\ Its relationship with dental implants and prosthodontics
}

\section{Sada-Garcalda, Vicente * Caffesse, Raúl G.}

*Práctica privada dedicada en exclusiva a la ortodoncia en Navarra.

**Profesor del Postgrado de Periodoncia Universidad Autónoma de Nuevo León, Monterrey,México

\section{Correspondencia}

Vicente Sada Garralda

Yangüas y Miranda $21,3^{\circ} \mathrm{Izda}$

31003 Pamplona, Navarra

Email.vsadag@terra.es
Resumen: Se ha expuesto un método de trabajo en el que la correcta preparación y planificación de los casos, permite obtener resultados predecibles a pesar de la complejidad de sus problemas. Favorece el entendimiento entre todos los profesionales de los distintos perfiles que integran el equipo rehabilitador, pues el remontaje sirve como herramienta para comunicar los objetivos que cada uno busca. Además, todas las referencias obtenidas de la fabricación del encerado, acortan el tiempo de las fases quirúrgica, ortodóncica y prostodóncica del tratamiento. Por otro lado, con los conocimientos obtenidos, se disminuyen los posibles riesgos.

Este método de trabajo es beneficioso para todos, pudiendo ofrecer al paciente un tratamiento mucho más satisfactorio.

Palabras clave: Pacientes adultos, Tratamiento multidisciplinario, Remontaje Ortodoncia, Rehabilitación.

Abstract: An approach to therapy has been described in which the correct preparation and detailed planning of the cases allow for the obtainment of predictable results in spite of the complexity of their problems. It favours the communication and understanding of all the specialists that integrate the treatment team. The treatment planning set-up is used as a tool to communicate the different objectives that each member seeks. Furthermore, all the information obtained during the wax-up will shorten the treatment time during the surgical, orthodontic, and prosthodontic phases. On the other hand, the knowledge achieved helps to minimize the possible risks to be encountered during these phases.

All the specialists will benefit with this working methodology, being able to offer a much more satisfactory treatment to the patient.

Key words: Adult patients, Multidisciplinary treatment, Treatment planning set-up, Orthodontics, Rehabilitation.

\begin{tabular}{ccc}
\hline Fecha recepción & Fecha última revisión & Fecha aceptación \\
$17-12-2003$ & $26-02-2004$ & $18-03-2004$ \\
\hline
\end{tabular}

BIBLID [1138-123X (2004)9:2; marzo-abril 125-256]

Sada-Garral V, Caffesse RG. Enfoque ortodóncico en el tratamiento multidisciplinario de pacientes adultos. Su relación con implantes y prostodoncia. RCOE 2004; $9(2): 195-207$. 


\section{Introducción}

Como se ha descrito en comunicaciones previas ${ }^{1,2}$, los pacientes adultos presentan una problemática muy compleja que debe ser tratada por un equipo multidisciplinar. Normalmente el tratamiento comienza restaurando las caries, endodoncias si se necesitan y tratamiento periodontal. Una vez eliminado el proceso inflamatorio gingival, debe continuarse con el trabajo por expertos en el áreas que corresponda según el caso.

Si los pacientes presentan maloclusión y no hay perdida de dientes, la siguiente fase sería realizar el tratamiento ortodóncico directamente; pero son la minoría, ya que normalmente presentan muchas perdidas dentales. En numerosos casos, el movimiento que se quiere hacer es imposible porque no hay dientes suficientes para utilizar como anclaje (fig. $1 \mathrm{~A}-\mathrm{F}$ ). Al tratar de mover unos dientes en una dirección, se produce el movimiento de los dientes de anclaje en el sentido contrario; un efecto indeseado la mayoría de las veces. Sería necesario un anclaje casi total, para poder hacer estos tratamientos con éxito.

Por otro lado, si la mejor forma de reemplazar los dientes perdidos, en estos pacientes, es utilizando implantes como soporte para las prótesis y se ha demostrado que son el anclaje ideal para hacer movimientos ortodóncicos (ya que una vez osteointegrados no se pueden desplazar-5), poniéndolos al principio, se podrán solucionar muchos casos que de otra forma sería imposible. Se podrán acercar, alejar, intruir y extruir los dientes con respecto a los implantes sin producir- les el más mínimo movimiento. De esta forma, los implantes tienen la doble finalidad de servir como anclaje para el tratamiento ortodóncico y además, como pilares para las prótesis definitivas ${ }^{6^{* *}}$.

Si se quieren poner los implantes antes de hacer el tratamiento ortodóncico, se debe hacer una planificación muy minuciosa de los movimientos que se piensan hacer y donde se pondrán las prótesis al final. Si hay un error en la posición de un implante, puede limitar el movimiento de algún diente 0 imposibilitar hacer una buena prótesis. La determinación de la posición de los implantes puede ser muy confusa en muchos casos antes de empezar el tratamiento ortodóncico, ya que se van a acercar o alejar los dientes contiguos. Para saber esa posición, se debe predeterminar el resultado final haciendo un remontaje en cera de todos los dientes, considerando también el tamaño y forma de las futuras coronas implantosoportadas ${ }^{7 *} \cdot$ (fig. $1 \mathrm{~J}$-Q).

Hay ocasiones en las que el sitio correcto donde se debe poner el implante, coincide con la posición de algún diente que posteriormente se va a desplazar. También puede darse el caso de que no haya espacio suficiente porque los dientes contiguos están inclinados hacia la zona desdentada. Cuando se dan estas situaciones, se debe comenzar con el tratamiento ortodóncico para desplazar los dientes y posibilitar la colocación de los implantes. Si se colocan pronto, se podrán incorporar como anclaje y servirán de ayuda para completar con éxito el tratamiento ortodóncico.

Siguiendo este método de trabajo, hay muchas áreas donde se necesitan hacer dos cirugías para implantarlos. En vez de esperar al final y poner los implantes de cada zona en una cirugía, se colocan antes de la ortodoncia aquellos que no coinciden con la situación de ningún diente y tienen espacio suficiente y después de mover aquellos dientes que estorban mediante ortodoncia, se pone la segunda tanda. También es posible encontrar que en la zona donde se debe poner el implante no hay suficiente hueso, siendo necesario regenerarlo, cuando muy cerca o con diferente angulación se podría colocar sin problemas. Así es que esta filosofía de trabajo puede ser más incómoda y agresiva para el paciente, pero es la única forma de conseguir un resultado predecible y que cumpla con los objetivos funcionales y estéticos que se persiguen en este tipo de tratamientos. De otra forma, se debe contar con que la suerte esté del lado de los diferentes especialistas.

\section{Planificación de un caso de ortodoncia con implantes u prólesis}

Debido a la duración y coste de este tipo de tratamientos, hay que dar al paciente la máxima información antes de comenzar a trabajar en su boca. Es más fácil que asimile todo y tendrá menos dudas de todas las implicaciones que conlleva, si se explica en varias citas. Además, de esta forma tendremos la ocasión de conocer mejor al paciente y podremos tomar decisiones más acertadas en caso de varios posibles planes de tratamiento, si 
alguno de ellos requiere más colaboración 0 va a ser más largo, etc. En muchas ocasiones, el paciente puede sentir que se está perdiendo el tiempo o que se va a alargar mucho y renuncian a seguir, así que estas primeras citas sirven como el primer filtro para aquellos que no cuentan con la paciencia o mentalización necesarias. Si después de varias citas y con toda la información de que dispone, el paciente quiere seguir adelante, es porque tiene verdadero interés, está muy mentalizado y con ganas de empezar a trabajar, requisitos indispensables para afrontar un tratamiento de este tipo.

La tabla 1 muestra el protocolo a seguir en la planificación del tratamiento.

En la primera visita se describe someramente cuál es la situación de la boca y se explica que son necesarios una serie de estudios para determinar cuales son los problemas y posibles planes de tratamiento. Es muy común que el día de la explicación quieran empezar inmediatamente y a los pocos días se arrepientan cuando piensan en la duración, coste y tiempo que deben dedicar. Para evitar tener que realizar estudios que no sirvan para nada y que roban mucho tiempo y dinero, no se deben realizar el primer día, sino que se deber dar al paciente una segunda cita para efectuarlo. De esta forma tiene tiempo para pensar si verdaderamente quiere hacerse el tratamiento.

Después de tomados los registros necesarios, se recopilan y se empieza a hacer un listado de los problemas, buscando una solución para cada uno de ellos, desarrollando así el plan o pla-

Tabla 1. Esquema del protocolo de planificación del tratamiento

\begin{tabular}{ll} 
VISITAS & \multicolumn{1}{c}{ TRABAJO A REALIZAR } \\
\hline Primera & - Describir brevemente el problema y sus consecuencias. \\
& - Explicar los pasos principales del tratamiento. \\
& - Planificar próximas visitas. \\
\hline Segunda & - Toma de estudios (RX-fotos y modelos). \\
Tercera & - Explicar diagnóstico general y posibles planes de trata- \\
& miento, con la decisión de si se hace o no remontaje. \\
& - Presentar tratamiento ortodoncico y su presupuesto. \\
& - Hacer una aproximación de implantes necesarios con pre- \\
& supuesto aproximado. \\
& - Enfatizar la necesidad de hacer remontaje, si es necesario, \\
& para presentar tratamiento y presupuesto definitivos. \\
& - Motivar al paciente a aceptar el plan propuesto. \\
Cuarta & - Enseñar y explicar remontaje. \\
& - Dar presupuesto de implantes y prótesis definitivo. \\
& - Dar citas para empezar.
\end{tabular}

nes de tratamiento. Con esta información, el ortodoncista deberá determinar la complejidad del caso desde el punto de vista multidisciplinario y así decidir si el tratamiento es complejo y requiere remontaje o es lo suficientemente sencillo como para poder ser planificado y realizado sin él (fig. 2 ).

Hasta este momento y ya en la tercera visita, sólo se puede dar el diagnóstico, plan de tratamiento provisional y presupuesto de ortodoncia pues todavía no se ha realizado el remontaje en cera de los dientes y futuras prótesis. Muchas veces no está claro cuántos implantes caben en una zona desdentada, así que hasta entonces no se sabrá el número exacto y de eso va a depender gran parte del tratamiento. Antes de empezar a hacer el remontaje hay que explicarle al paciente que hacerlo requiere muchas horas de laboratorio y que sólo merece la pena realizarlo si está seguro de seguir adelante. Antes de tomar esta decisión, ya se le ha dado una aproximación del coste y duración para que pueda meditarlo. Si acepta, le damos una nueva cita para enseñárselo y mientras, el odontólogo o estomatólogo, endodoncista o periodoncista, pueden seguir realizando, en caso de no estar terminados, sus tratamientos.

En la cuarta cita se facilita al paciente el plan de tratamiento definitivo, con la cantidad exacta de implantes y se le enseña la predicción del resultado final. También se conocerá cuál es el presupuesto definitivo de los implantes y las prótesis. Lo más probable es que acepte las condiciones y continúe con el tratamiento, dado que anteriormente ya se le había facilitado casi toda la información.

En la siguiente cita se empezará el tratamiento, pues tanto el paciente como los diferentes profesionales que intervendrán saben perfectamente 10 que tienen que hacer. 


\section{Consideraciones}

\section{que se deben tener}

\section{en cuenta}

\section{antes de realizar}

\section{el remontaje}

A la hora de hacer el remontaje, se debe empezar por determinar qué dientes van a permanecer en la boca, pues muchos tendrán un pronóstico dudoso. Para tomar esta decisión, hay que tener en cuenta que el objetivo final en este tipo de tratamientos es conseguir un resultado muy equilibrado tanto oclusal como estético y además, que al poner dientes implantosoportados, se disminuyen las cargas a los dientes naturales.

Considerando estos factores, se puede ser muy conservador y procurar mantener todos los dientes que se pueda, aunque el pronóstico sea dudoso (fig. 1-F). Es sorprendente como dientes con gran pérdida de hueso y movilidad, recuperan su salud y se estabilizan al devolverles una mejor función, disminuyendo sus cargas o eliminando sus prematuridades. Anteriormente se pensaba que mantener dientes con pronóstico dudoso, aceleraría la destrucción del periodonto interproximal adyacente y se extraían, provocando una constricción del hueso en esa zona. Estudios de seguimiento recientes indican que estos dientes no afectan significativamente el periodonto interproximal después del tratamiento. La recomendación actual es retener en la boca estos dientes periodontalmente afectados, mientras la lesión pueda mantenerse controlada $a^{9.12}$.

Gracias a la predicción, se sabe cuál va a ser el tamaño de las coronas y su localización exacta, por lo que se tienen muchas más y se reduce notablemente el tiempo de tratamiento (fig. $1 \mathrm{~N}-0$ ). En definitiva, utilizando este método de trabajo, se reducen las consecuencias negativas que la ortodoncia puede tener en los dientes de pronóstico dudoso.

A la hora de planear los futuros movimientos, hay que considerar todos los beneficios que estos pueden ofrecer para mejorar la salud de los tejidos blandos y de hueso alveolar que se han descrito en las comunicaciones previas $^{1,2}$. Los implantes pueden ser utilizados también como anclaje para la terapia ortodóncica. Así, se pueden planificar objetivos muy ambiciosos, que son impensables, si se quieren realizar anclándose sobre dientes naturales, como la intrusión de molares extruidos que no dejan altura para rehabilitar su antagonista (fig. 3). Si se quisiera intruir un molar apoyándose en un diente contiguo, se produciría más extrusión de éste último, que intrusión del molar. Con la ayuda de un implante cercano, se puede realizar la intrusión simplemente poniendo los brackets a diferentes altura, ya que como el implante no se mueve, toda la fuerza repercute en el diente. Cuando no hay implante al lado, también se puede intruir un molar extruido, poniendo un implante en el lugar de su antagonista y mediante imanes colocados uno en el implante y otro en el molar de forma que se repelan. De este modo se consigue una fuerza suflciente como para intruirlo varios millmetros en unos meses (fig 3).

\section{Pasos para hacer el remontaje}

Como se ha dicho anteriormente, hacer el remontaje implica un trabajo que requiere muchas horas en el laboratorio y que puede parecer innecesario, pero lo cierto es que cuanto más se prepare el caso, más fácil resultará el trabajo en la boca y mejor será el resultado final.

El primer paso comienza duplicando fielmente los modelos originales al menos dos veces. Para ello, lo ideal es utilizar la máquina de moldeado a presión Biostar o Ministar (Scheu-Dental) con láminas blandas de protectores bucales de $3 \mathrm{~mm}$ de grosor (Bioplast, Scheu-Dental). Antes de duplicarlos, es necesario poner unas referencias en el paladar y suelo de la boca, que se hacen colocando unas gotas de silicona autoadheribles. Un juego de modelos duplicados se utiliza para cortar los dientes y reubicarlos junto con los implantes en su posición óptima. Las referencias de silicona se utilizan para transferir esta nueva posición al modelo original en maloclusión ${ }^{8}$.

Según lo complejo que sea el caso y la cantidad de dientes que se van a recolocar, será necesario montar uno de los juegos en articulador semiajustable. Se puede hacer sobre modelos sin montar en un articulador si no se modifican los molares y se mantiene la dimensión vertical inalterada, pero se deben utilizar modelos perfectamente recortados para que sea posible su manejo.

Antes de cortar cada diente, se deben hacer marcas de referencia para saber posteriormente cuánto se mueve cada uno y se empiezan a cortar y 


\section{Figura 1. Caso 1}
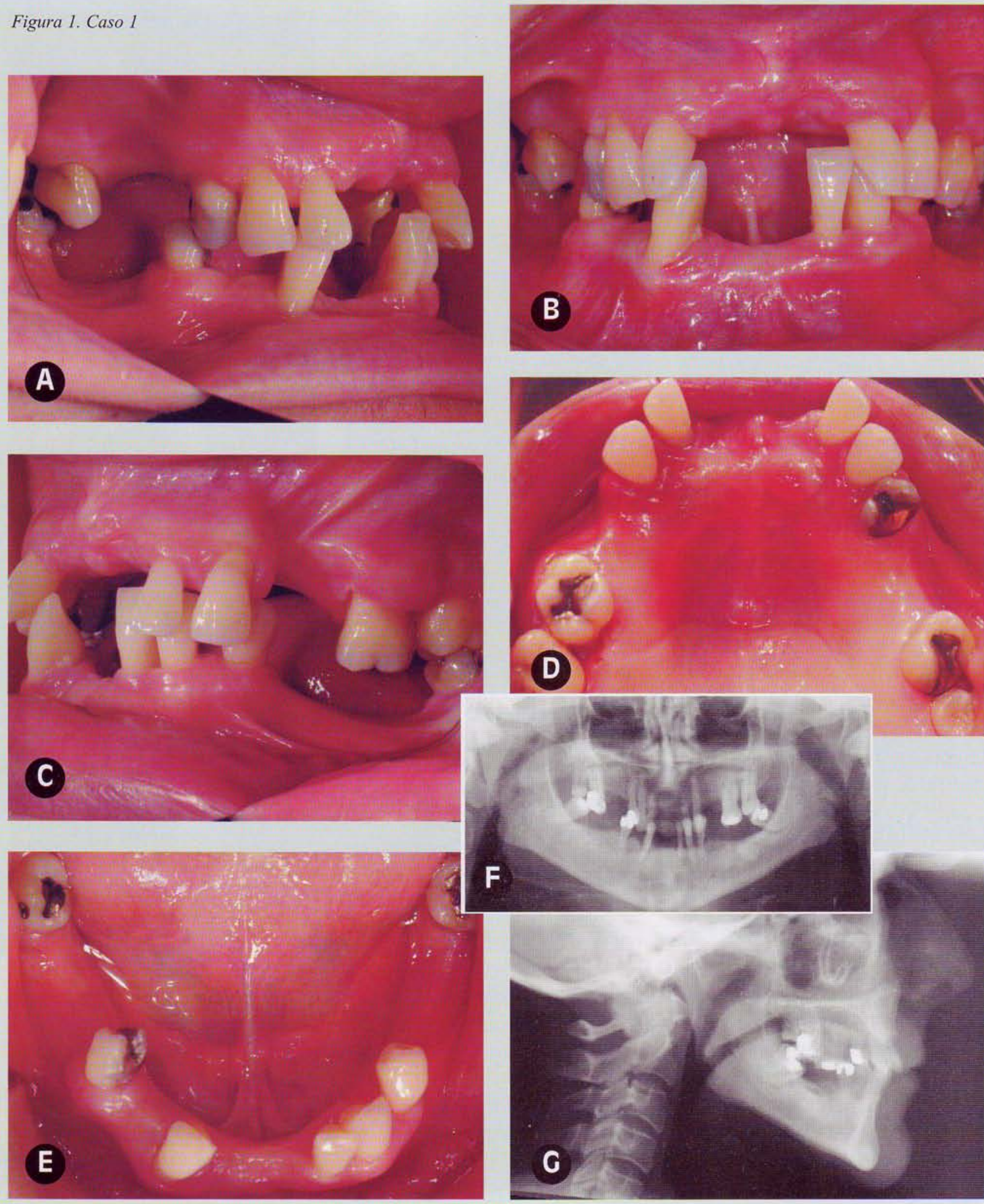

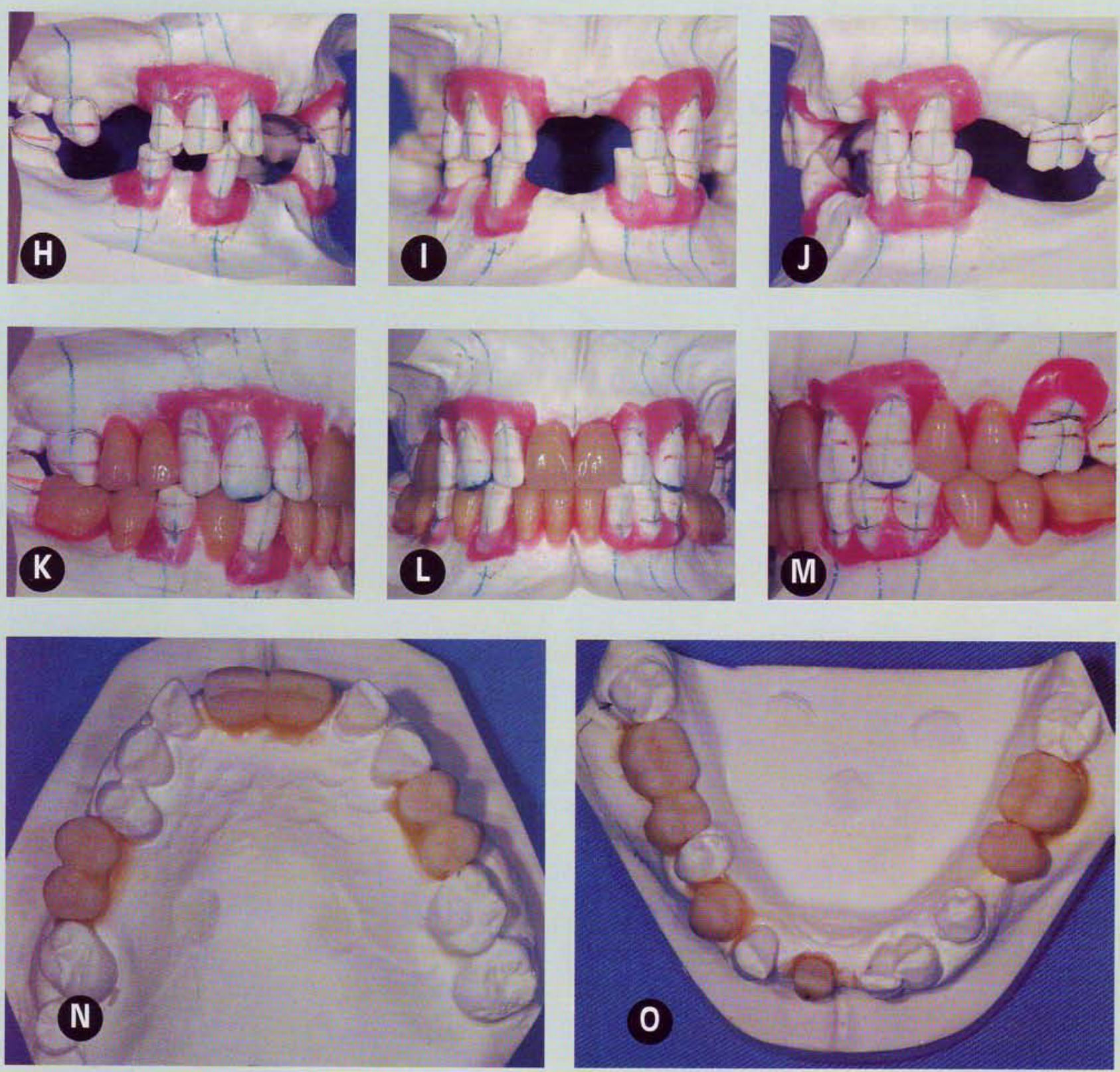

recolocar según los objetivos preestablecidos (fig. $1 \mathrm{H}-\mathrm{J}$ ). El odontoestomatólogo que se dedique a la práctica exclusiva de la ortodoncia es quien, personalmente, tiene que acabar de completar esta nueva colocación, pues se necesitan conocimientos de las limitaciones que se pueden encontrar a la hora de mover los dientes. Además, haciéndolo va a obtener información muy importante que luego le va a servir durante el tratamiento.

Además de recolocar en una buena posición los dientes existentes, se deben establecer los espacios donde posteriormente se van a poner próte- sis, reproduciendo lo más fielmente posible el tamaño y forma de los dientes naturales. Muchas veces es preferible sustituir un molar perdido por un premolar, porque el tamaño de un implante de molar sería muy ancho para una zona donde se ha perdido hueso. El dentista rehabilitador debe con- 

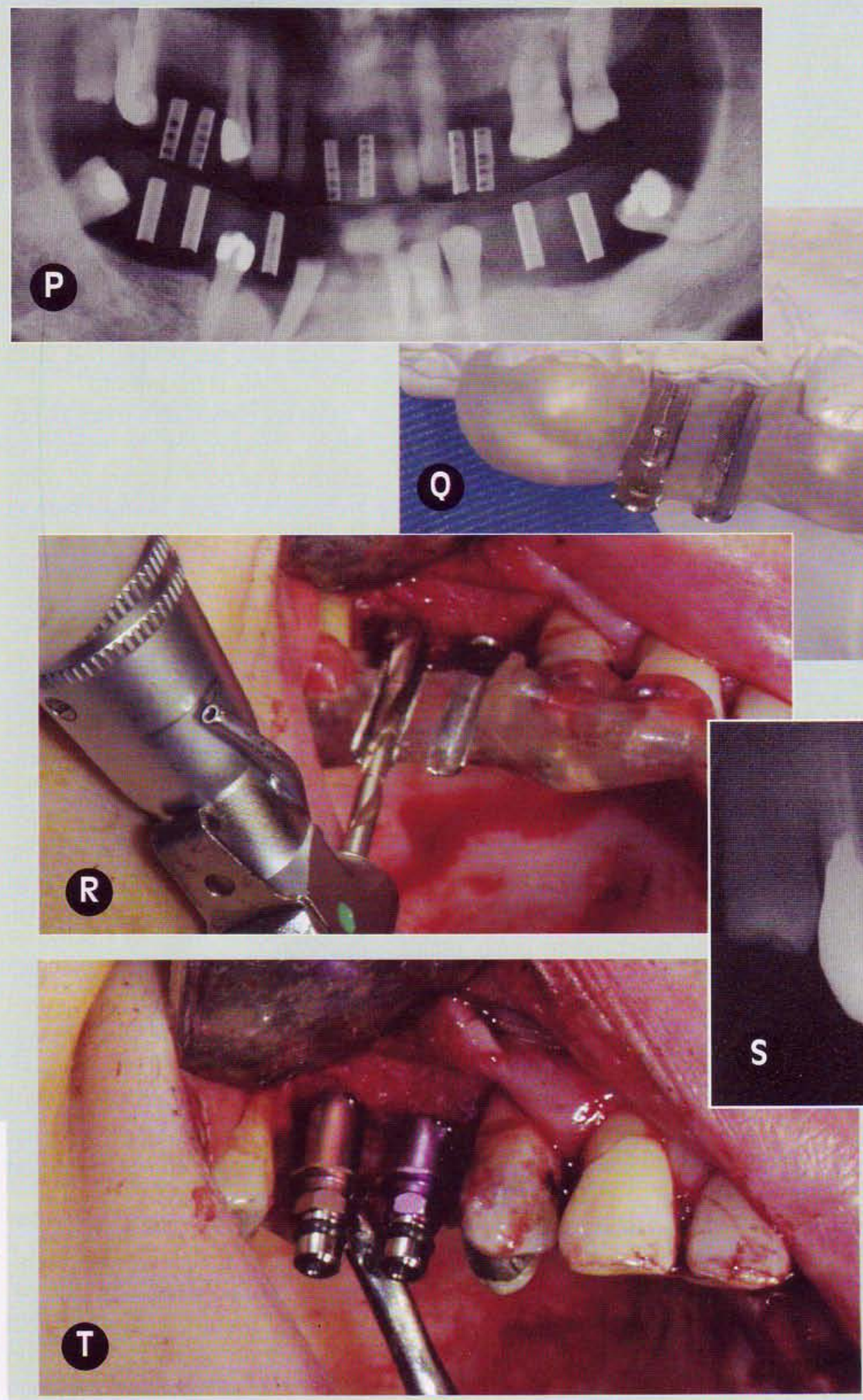

El tratamiento periodontal $y$ los implantes de la figura $I$ han sido realizados por el Dr. Francisco Sada-Garralda. 
firmar que el remontaje ortodóncico cumple estos requisitos protésicos, antes de empezar con el encerado de los dientes que faltan. Este encerado va a servir para determinar la posición de los implantes, fabricar las restauraciones provisionales necesarias en la ortodoncia y también para la realización de las prótesis definitivas (fig. 1 K-M).

La localización de los implantes se tiene que transferir ahora al segundo juego de modelos duplicados donde tenemos la posición original de los dientes. En este momento la disposición de modelos exactamente iguales es imprescindible; de otra forma no se podrán traspasar fielmente las relaciones establecidas. Mediante este paso, se puede ver si algún diente mal colocado está en el sitio donde se quiere poner un implante, en cuyo caso deberá postergarse su colocación hasta que el diente se haya desplazado ortodóncicamente (fig. $1 \mathrm{~N}$ $0)$.

Después de transferida toda la información del remontaje al modelo original, sólo falta llevarla a la boca del paciente. Todo el trabajo realizado anteriormente, culmina con la fabricación de una guía quirúrgica que permite al cirujano colocar los implantes exactamente en la posición que se ha determinado como ideal (fig. 1 Q-T). De otro modo, la intervención es más tensa para el cirujano que tiene que ir improvisando mientras controla todos los riesgos y puede restar importancia a pequeños detalles que pueden imposibilitar posteriormente hacer una buena prótesis. Antes del día de la cirugía, se deben tomar radiografías de las zonas donde se planea colocar implantes o una panorámica con las guí- as quirúrgicas puestas para comprobar si todo está correcto en la predicción y se pueden tomar como referencia fiable (fig.1-P). Siguiendo todos los pasos correctamente, se podrá determinar la longitud, grosor, localización y angulación de los implantes, además de cuántos podemos colocar y las distancias con puntos peligrosos a los que el cirujano debe evitar acercarse.

Aquí termina la fase de diagnóstico y planificación y comienza la fase de trabajo en la boca, que a pesar de seguir siendo difícil y expuesta a cambios, siempre será más predecible y con mejores resultados que improvisando.

\section{Comienzo del iratamiento}

Esta fase comienza con la colocación de los implantes que no coinciden con la posición actual de algún diente, antes de la ortodoncia. La guía quirúrgica va a ser la referencia imprescindible para hacerlo bien. Al terminar de colocarlos, será necesario hacer una radiografía panorámica con la guía puesta para ver si se han posicionado exactamente como estaba previsto 0 ha habido algún pequeño cambio que debe ser considerado al hacer las prótesis.

Después de los meses necesarios para su osteointegración, se les carga la prótesis provisional exactamente igual a la que se había previsto en el remontaje y es el momento ideal para empezar el tratamiento de ortodoncia. En la boca están los dientes naturales en malposición, junto con los nuevos dientes implantosoportados en el lugar, tamaño y anatomía que se ha determinado como ideal para el caso. De esta forma, las prótesis sirven como referencia para saber hasta dónde hay que mover los dientes y además como anclaje para moverlos. Así pues, el tratamiento resulta mucho más sencillo, corto y en definitiva menos traumático para los dientes naturales.

Conforme se vayan eliminando los obstáculos durante el tratamiento, se irán poniendo los implantes que no se han podido colocar al principio. Cuanto antes esto sea posible, más útiles serán durante el tratamiento.

Una vez conseguidos todos los objetivos ortodóncicos, se retiran los aparatos y se pone la retención como en todos los casos. Normalmente es recomendable esperar al menos seis meses para que los dientes se estabilicen algo, antes de empezar a sustituir las prótesis provisionales por las definitivas. Utilizando este sistema de trabajo, la fabricación de ésta es mucho más sencilla y rápida. Si se utiliza para la confección de la prótesis definitiva la forma de los dientes del remontaje, la sustitución se puede hacer antes de los seis meses pues no hay que hacer tantas pruebas en el laboratorio y no se dejan los dientes sin retención.

Los tres casos que se presentan, muestran los resultados que se pueden obtener coordinando correctamente los tratamientos ortodóncico, implantológico y prostodóncico en adultos con presencia de zonas desdentadas y con malposición dental.

Caso clínico 1. Paciente de sexo femenino de 48 años de edad con periodontitis severa, edentulismo parcial y malposición dentaria. Ha recibido tratamiento periodontal, incluyendo 
cirugías. La enfermedad periodontal está controlada y lleva un mantenimiento rutinario y riguroso. Los tejidos periodontales muestran estado de salud y la paciente practica una higiene oral aceptable, tal como se demuestra en las imágenes preortodoncia (fig.1 A-E). Debido a estos buenos resultados, se decidió mantener todas los dientes presentes a pesar de contar con pérdida ósea avanzada, con defectos angulares y signos de trauma oclusal (fig. 1-F). En la radiografía lateral se comprueba la inclinación de los incisivos superiores e indica que a pesar de existir espacios sin dientes, no se debe retruir el sector anterior ya que ello afectaría negativamente el perfil estético de la paciente. Esta toma radiográfica ofrece información muy importante para la restauración del sector anterior que debería ser tenida en cuenta tanto para la colocación de implantes como para la rehabilitación protésica (fig. 1-G). Una vez duplicados, los modelos de diagnóstico fueron utilizados para cortar los dientes existentes y reubicarlos en la posición que se consideró óptima (fig. $1 \mathrm{H}-\mathrm{J}$ ). A continuación, se enceraron las piezas a ser reemplazadas protésicamente, determinando así la cantidad de implantes y posición en que debían ser colocados (fig. 1 K-M). Toda esta información obtenida, es transferida a los modelos de trabajo, con el objeto de construir la guía quirúrgica que la llevará a la boca (fig. 1 $\mathrm{N}-\mathrm{O}$ ). Es necesario comparar los encerados de estas figuras, con las figuras $1 \mathrm{D}$ y E. Así se puede comprobar el tratamiento propuesto y la modificación en la posición de los dientes. El encerado de diagnóstico provee información muy importante para la planificación del tratamiento. Así por ejemplo, observando el maxilar inferior, se comprueba que no existe lugar suficiente para colocar las piezas 35,31 y 42 a menos que se desplacen los dientes contiguos. Por ende, los implantes correspondientes deberán ser colocados durante el tratamiento ortodóncico. Antes de la colocación de los implantes previos a la ortodon$\mathrm{cia}$, hay que tomar una radiografía panorámica con la guía quirúrgica en la boca para comprobar si su fabricación es correcta o hay que tomar alguna precaución extra para no dañar ningún diente contiguo ( fig. 1-P). En ésta, también se podrá ver la longitud que deben tener los implantes para no entrar en los senos maxilares o tocar nervio alguno. La guía quirúrgica con sus tubos guía para la colocación de los implantes, (fig. 1-Q) permitió colocar los mismos en la posición deseada y con la angulación correcta (fig. 1 R-T).

Caso clínico 2. Paciente de sexo femenino de 45 años de edad con varias pérdidas dentarias, enfermedad periodontal moderada, y maloclusión con apiñamiento, La paciente no presentaba ningún factor de importancia en su historia médica y al momento de la consulta, había ya dejado de fumar. Las figuras 2 A-E presentan la imagen clínica inicial y la figura $2 \mathrm{~F} \mathrm{la}$ imagen radiográfica. Se completó el tratamiento periodontal en base a raspaje y alisamiento radicular e higiene oral. Estando la paciente decidida a proseguir con su tratamiento restaurador, comenzó sus citas con el ortodoncista, quien determinó que el caso a pesar de su complejidad, era lo suficientemente claro como para no requerir remontaje. Se necesitaban tres implantes y sólo dos se podían poner en un principio. En el maxilar superior se colocaron dos implantes osteointegrados y fueron usados al comenzar el tratamiento de ortodoncia, seis meses después, como anclaje. Esto permitió distalizar el molar superior derecho y de esa manera crear espacio para colocar un segundo implante en ese cuadrante. De modo que los implantes fueron utilizados como anclaje ortodóncico primero y como pilares para la restauración fija, posteriormente. En el maxilar superior, se redujeron las coronas clínicas con el objeto de mejorar la proporción corona-raíz clínica de los incisivos centrales y laterales y se realizó stripping de canino a canino, para permitir la alineación del sector anterior. En el maxilar inferior se practicó stripping de molar a molar en una sola sesión y se activaron los dientes con un arco continuo y fuerzas muy ligeras. Se logró un espacio de aproximadamente $5 \mathrm{~mm}$ para corregir el apiñamiento anterior. Las figuras $2 \mathrm{C}$-L muestran el resultado obtenido después del tratamiento multidisciplinario. Obsérvese la oclusión lograda, el estado de salud periodontal y las restauraciones independientes implantosoportadas. Este caso demuestra claramente como una situación compleja de resolver, puede simplificarse si se planea adecuadamente y tomando en consideración la contribución de un tratamiento multidisciplinario. Comenzó con periodoncia e implantes, continuó con ortodoncia, realizando stripping para evitar extracciones y finalizó con odontología restauradora, a lo largo de un tratamiento que duró 14 meses. 


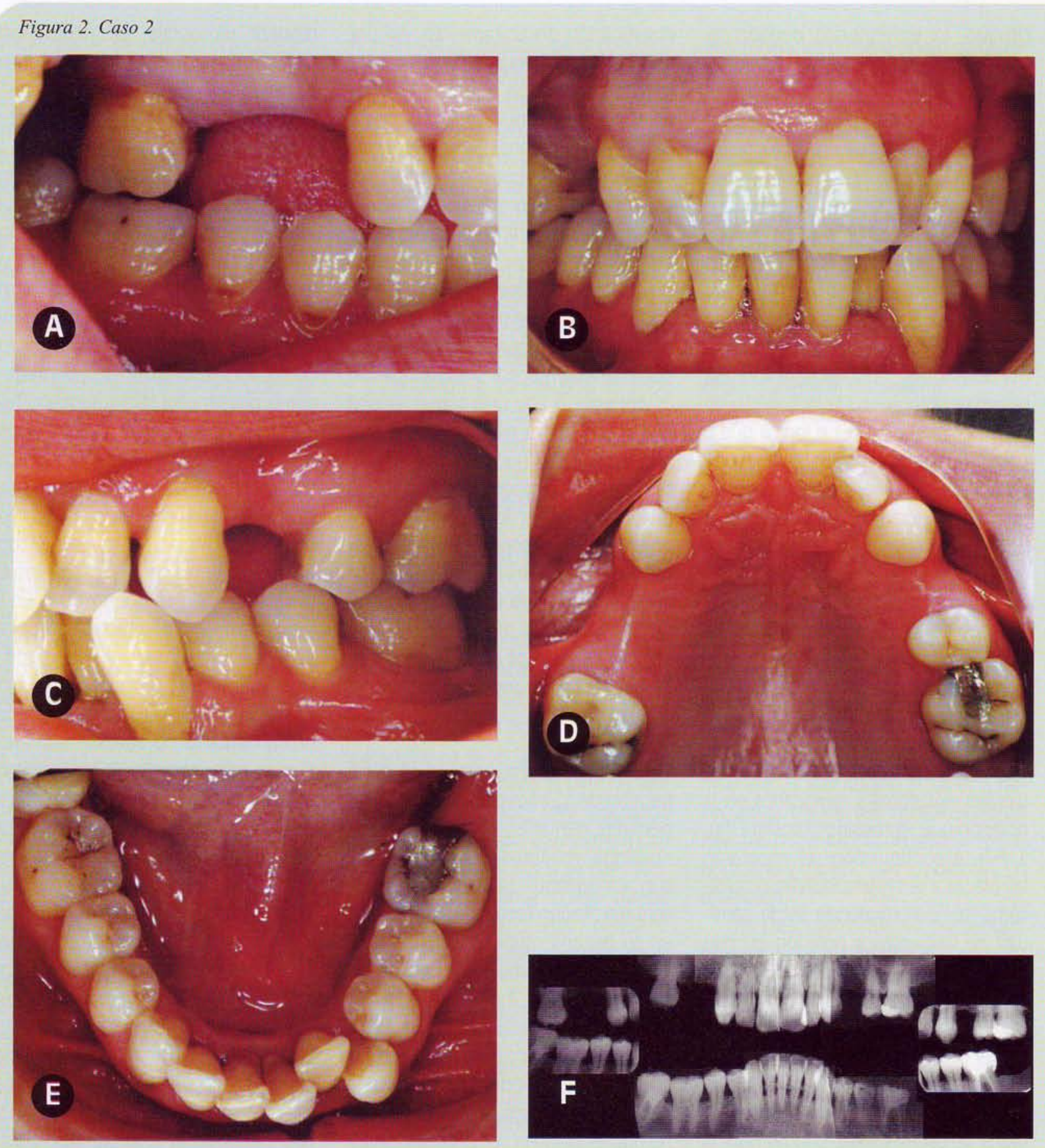

Caso clínico 3. Paciente de sexo femenino de 34 años de edad que acudió a la consulta con el objeto de reemplazar el primer y segundo molar inferior izquierdo que habían sido extraidos muchos años atrás. Además de presentar maloclusión, el primer molar superior izquierdo se había ex- truido por falta de antagonista, hasta prácticamente llegar a contactar con el reborde inferior (fig. 3-A). La salud periodontal era buena, por lo tanto no 

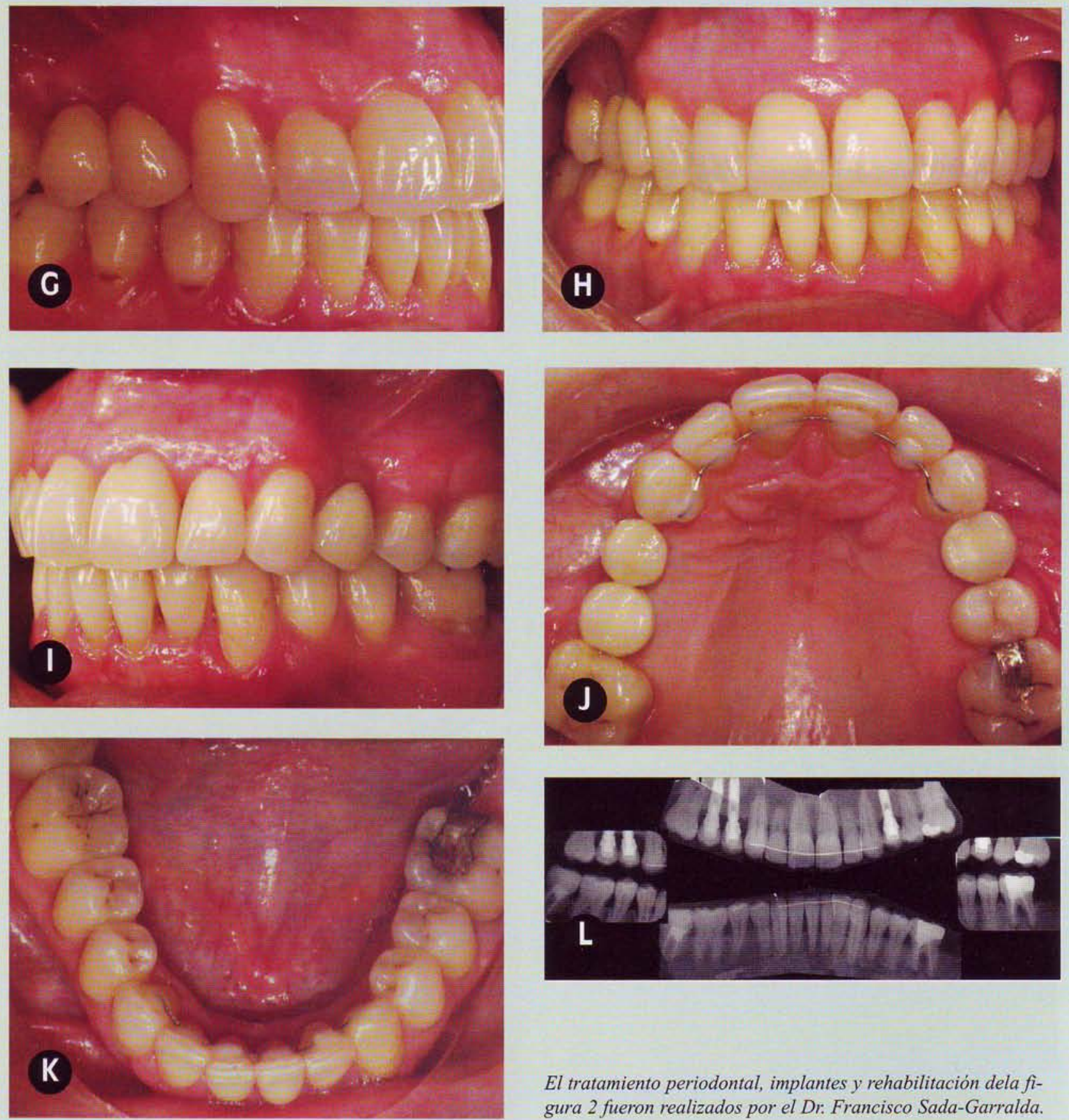

El tratamiento periodontal, implantes y rehabilitación dela figura 2 fueron realizados por el Dr: Francisco Sada-Garralda.

recibió más que una profilaxis y enseñanza de higiene oral. Fue determinado que para poder restaurar el área desdentada, era necesario corregir la maloclusión. Planeado el tratamiento, se colocaron dos implantes en el sector de 36 y 37 . Una vez osteointegrados, se expusieron y en sus tapas se cementaron pequeños imanes. Del mismo modo, se colocaron otros imanes en la cara oclusal del molar extruido. Todos enfrentados del mismo po- 
Figura 3. Caso 3
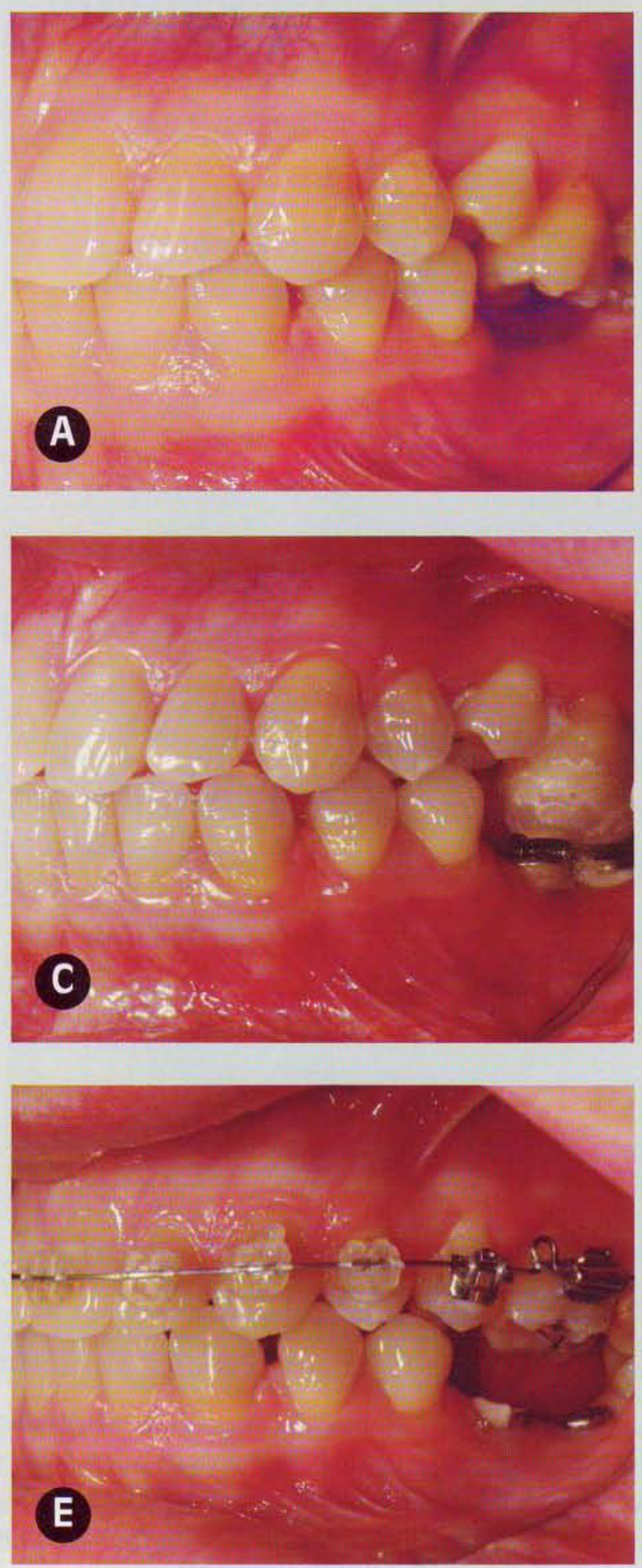
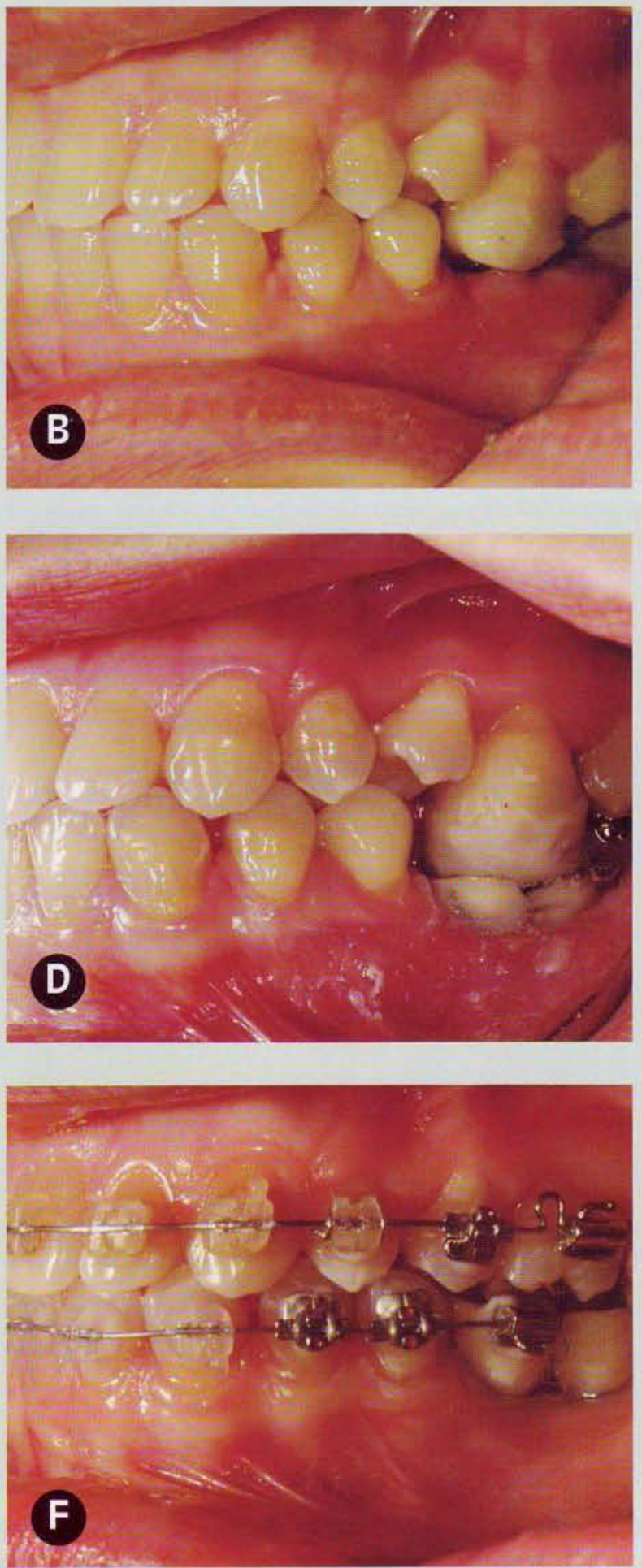

Los implantes y la rehabilitación de la figura 3 está siendo realizada por el Dr: Francisco Sada-Garralda. 
lo, contactaban generando una fuerza de rechazo (fig. 3-B). Tres meses más tarde, el molar superior mostraba ya intrusión y las caras de los imanes estaban separadas (fig. 3-C). Cuando esto sucede, la fuerza de rechazo dis- minuye hasta llegar a anularse. Es necesario moverlos y recementarlos de modo que vuelvan a estar en contacto otra vez (fig. 3-D). Nueve meses después de iniciado el procedimiento, el molar superior había sufrido una in- trusión que lo llevo prácticamente al plano oclusal normal. Esto permitió la colocación de aparatología ortodóncica fija en el arco inferior, después de restaurar temporalmente los implantes (fig. $3 \mathrm{E}-\mathrm{F}$ ).

\section{Bibliografla recomendada}

Para profundizar en la lectura de este tema, el/los autor/es considera/an interesantes los artículos que aparecen señalados del siguiente modo: *de interés * ${ }^{*}$ de especial interés.

1. Sada-Garralda V, Caffesse RG. Enfoque ortodóncico en el tratamiento multidisciplinario de pacientes adultos. Su relacion con la periodoncia $\mathrm{RCOE}$ 2003;8(6):673-84

2. Sada-Garralda, V. Caffesse, RG. Enfoque ortodóncico en el tratamiento multidisciplinario de pacientes adultos. El stripping y sus efectos sobre dientes y periodonto. RCOE 2004; 9(2):179-89.

3. Smalley WM, Shapiro PA, Hohl TH y cols. Osseointegrated titanium implants for maxillofacial protraction in monkeys. Am J Orthod Dentofac Orthop 1998;84: 285-95.

4. Roberts W, Smith R, Ziberman Y y cols. Osseous adaptation to continuous loading of rigid endosseous implants. Am J Orthod 1984;86:95-111.

5. Roberts W, Helm F, Marshall K y cols. Rigid endosseous implants for orthodontic and orthopedic anchorage. Angle Orthod 1989:59:24755
$6^{* *}$. Kokich VG. Managing complex orthodontic problems: The use of implants for anchorage. Semin Orthod 1996;2:153-60

El autor describe el momento, secuencia y método para establecer la localización adecuada de los implantes. Demuestra con varios casos, lo importante que es la comunicación interdisciplinaria y planificación con un remontaje de diagnóstico.

7**. Smalley WM. Implants for tooth movement: Determining implant location and orientation. J Esthet Dent 1995;7:62-72.

En este trabajo se describe una técnica de laboratorio para predecir la mejor posición de los implantes, considerando los movimientos ortodóncicos previstos y las necesidades estéticas para que la prótesis final quede lo mejor posible. Además, este método permite fabricar la guía quirúrgica para trasladar esta información de los modelos a la boca.
8. Smalley WM, Blanco A. Implants for tooth movement: A fabrication and placement technique for provisional restorations. J Esthet Dent 1995;7:150-4.

9. Chance R, Low SB. Survival characteristics of periodontally-involved teeth: A 40-year study. J Periodontol 1993;64:701-5

10. Klock KS, Haugejorden O. In vitro determination of the forceps level for extraction of teeth for periodontal reasons. J Clin Periodontol 1993;20:155-60

11. DeVore CH, Beck FM, Horton JE. Reteined «hopeless» teeth. Effects on the proximal periodontium of adyacent teeth. J Periodontol 1988;59:647-51.

12. Wojcik MS, DeVore CH, Beck FM y cols. Reteined "hopeless" teeth: Lack of effect periodontally-treated teeth have on the proximal periodontium of adyacent teeth 8-years later. J Periodontol 1992;63;663-6. 